\title{
Liver Registration for the Follow-Up of Hepatic Tumors
}

\author{
Arnaud Charnoz ${ }^{1,3}$, Vincent Agnus ${ }^{1}$, Grégoire Malandain ${ }^{2}$, Clément Forest $^{1}$, \\ Mohamed Tajine ${ }^{3}$, and Luc Soler ${ }^{1}$ \\ ${ }^{1}$ IRCAD R\&D, Strasbourg, France \\ ${ }^{2}$ Epidaure Research group, INRIA, Sophia Antipolis, France \\ ${ }^{3}$ LSIIT, CNRS/ULP, Strasbourg, France
}

\begin{abstract}
In this paper we propose a new two step method to register the liver from two acquisitions. This registration helps experts to make an intra-patient follow-up for hepatic tumors.

Firstly, an original and efficient tree matching is applied on different segmentations of the vascular system of a single patient 1 . These vascular systems are segmented from CT-scan images acquired (every six months) during disease treatement, and then modeled as trees. Our method matches common bifurcations and vessels. Secondly, an estimation of liver deformation is computed from the results of the first step.

This approach is validated on a large synthetic database containing cases with various deformation and segmentation problems. In each case, after the registration process, the liver recovery is very accurate (around $95 \%)$ and the mean localization error for 3D landmarks in liver is small (around $4 \mathrm{~mm}$ ).
\end{abstract}

\section{Introduction}

Motivations: Liver Tumors Follow-Up: The main purpose of our work is to make an intra-patient follow-up of tumors (see our previous work [2]). This task is difficult since the liver is a highly deformable organ. Thus, tumor matching relies on the liver deformation. To estimate this deformation, we propose to compute a deformation field from reliable landmarks and then extrapolate it to a dense field. It is a well-known result that the most reliable landmarks to estimate deformations sustained by the liver are provided by its vascular network [3 4 4 5 : 6 6 7. We use our iterative tree matching algorithm on the vascular system, to match common bifurcations and edges (see [1 for more details). Thus, each match provides a displacement vector. From this sparse data a dense deformation field is built.

Proposal: The remainder of this paper is organized as follows. We briefly recall related methods to solve the problematics of our approach to compare and to justify our approach. Then, we summarize our iterative oriented tree matching (detailed in 1]). The next part describes the registration algorithm. The last section deals with the validation protocol and demonstrates the efficiency of this global approach (localization error reduced from $20 \mathrm{~mm}$ to $4 \mathrm{~mm}$ ). 


\section{Related Works}

Matching: Related works propose algorithms to match and/or register vascular systems (brain, liver and, in a similar manner, lung airways). Generally, veins are modeled as graphs computed from segmented images and skeletons 8 . Some authors use tree structure notions in their algorithms to register a tree with an image [3] or two trees [4]. Other approaches match structures (nodes and vessels), but use general graph matching methods [569] or specific methods like subtree isomorphism [7] which do not take segmentation problems into account.

The oriented tree matching problem is more specific than graph matching because the structure is oriented and the path that connects two nodes is unique. Moreover, it cannot be considered as an oriented subtree isomorphism problem because of the segmentation errors. Indeed, the segmentation process can miss some vessels (edges). This implies a (virtual) pruning on both trees (for example an edge in a tree could be represented by several successive edges on the other tree) and thus the tree topology differs between acquisitions.

In our previous work [1, vascular systems are modeled as trees. Then, tree vertices are matched using a cost function that takes possible segmentation errors into account. Our algorithm does not focus on the best solution (given two edge sets to match) but on the most likely solutions which are updated during the process.

Vector Field Interpolation: Several methods exist to produce a deformation vector field from sparse displacements : the method in [10] is based on the computation of the optical flow. Another approach uses krigging [11] or considers the deformation field as a derivate of a potential scalar map modeled by Green's function [12. A generalization of scalar splines to vector interpolation is proposed in 13 to estimate displacements.

Our first time method is simpler and less computive consuming than previously cited ones. Indeed liver deformations are less complicated than generalized flow fields (no vortex for instance). Our method is decribed in section 4 and its efficiency which is encouraging is discussed in section 5.

\section{Iterative Tree Matching Technique}

This section describes how we build correspondences among common structures in the vascular system. Skeletons computed from segmented vascular systems can be represented as oriented trees. The orientation symbolizes blood circulation flow. Nodes represent bifurcations and edges correspond to vessels between two bifurcations. Furthermore in our algorithm, some geometric attributes are added to the vessels (3D positions, radius, path).

Vascular trees segmented for a patient follow-up represent the same vascular system and our goal is to find common bifurcations and to register them. However, their topology and 3D positions may differ due to segmentation errors and deformations applied to them. The main challenge consists in using tree topology 

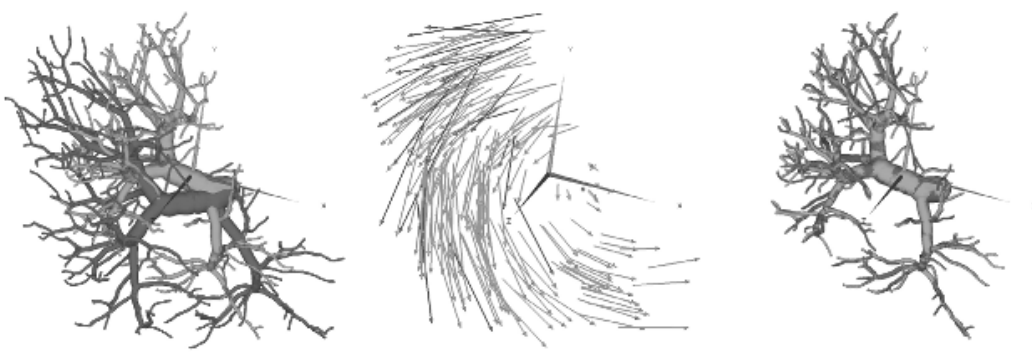

Fig. 1. [Left] A large deformation case is pruned at 20\% (\% of branch area randomly removed in both trees). [Center] The figure shows the result of our oriented tree matching, good matches are represented by light gray arrows and represent $91 \%$ of all nodes and wrong matches by dark gray arrows. [Right] The figure shows the tree registration after the process.

to detect deformations, and in parallel, geometric information to detect topology problems.

The idea of our algorithm [1] is to search for the best tree matching starting from roots (vascular system entrance). Since possibilities are numerous, we propose to generate and select the most relevant solutions. The algorithm starts by studying the root match and updates selected solutions while it explores and finds other possible matches in both trees. This means that some solutions selected at a given process step can be eliminated later if they become less relevant. The relevance of the solutions is evaluated at each step using a quality match criteria. We show in [1] that this algorithm matches $90 \%$ of common nodes on standard deformation and pruning. An example is shown in Fig. 1 and other results are shown on Tab. 2. For more information on this topic, please refer to our previous paper [1] in which this method is described and validated.

\section{Registration}

The previous step provides us with a match set which represents a deformation vector field. We explain here how we extrapolate it to a dense field in order to predict the liver deformation.

The matching provides us with a vector deformation $\boldsymbol{T}_{\boldsymbol{i}}=P_{i}^{\prime}-P_{i}$ in each correspondence point (bifurcation) $\left(P_{i}^{\prime}, P_{i}\right)$. Our method extrapolates this vector flow to yield deformation vectors in each point inside the liver. To compute the deformation $\boldsymbol{T}_{\boldsymbol{M}}$, we use Voronoi cells $V_{i}=\left\{P \in \mathcal{R}^{3}:\left\|P-P_{i}\right\| \leq\left\|P-P_{j}\right\|\right.$, $\forall j \neq i\}$. The extrapolated deformation $\boldsymbol{T}_{\boldsymbol{M}}$ in a point $M \neq P_{i}$ is defined by :

$$
\boldsymbol{T}_{\boldsymbol{M}}=\frac{1}{\operatorname{volume}\left(S_{M}\right)} \times \sum_{i} \operatorname{volume}\left(V_{i} \cap S_{M}\right) \times \boldsymbol{T}_{\boldsymbol{i}}
$$

where $S_{M}$ is a sphere centered on $M$ and of radius $d=\min _{i}\left\|M-P_{i}\right\|$. This deformation is a linear combination of surrounding bifurcation displacements. The impact of each displacement is correlated with its influence zone $\left(V_{i} \cap S_{M}\right)$. 

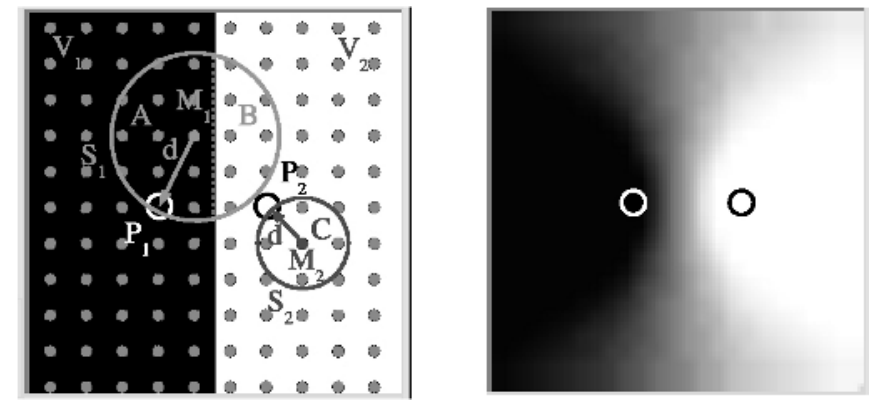

Fig. 2. [Left] The figure shows two color points $P_{1}$ (black) and $P_{2}$ (white), their associated Voronoi cell $V_{1}$ and $V_{2}$. The circles $S_{i}$ are defined using the distance $d$ from $M_{i}$ to the closest point $P_{i}$. [Right] The figure shows the result of our color interpolation.

To speed up the process, the displacement field is computed on a regular subsampled liver. Then, we use a trilinear interpolation to extend the results to whole liver. A $2 \mathrm{D}$ example with color interpolation of two points (white and black) is illustrated on Fig. 2.

\section{$5 \quad$ Experiments and Validation}

To validate this registration algorithm, it is necessary to have a large patient database. However, we lack multi-acquisitions of the same patient mostly because the time between two acquisitions is long as it is imposed by the disease treatment (around 6 months). This is why our real patient database is not sufficient to provide a clinical validation of our method. However, a real case is studied at the end of this paper and results are encouraging for the future.

At the moment, we have tested our algorithm on a large synthetic database. Even if synthetic cases differ slightly from real cases (deformations and segmentation problems are simulated), working with a synthetic database has some advantages. It allows to test many configurations and to have a gold standard, so that we can estimate the algorithm efficiency. In this section, we present how we build this database and obtain our results.

\subsection{Creating Virtual Patient}

To test and validate our algorithm, we worked on synthetic deformation applied on a liver and its hepatic vascular system. The liver model has been extracted from the Visible Man image which voxel resolution is $0.33 \times 0.33 \times 1 \mathrm{~mm}$ (cf. The Visible Human Project of NLM) with a segmentation that provides us an accurate quality model.

To simulate deformations, we use the minimally invasive hepatic surgery simulator prototype developed at INRIA [14]. This simulator provides a realistic deformation model for the liver and its vascular system. It uses complex biomechanical models, based on linear elasticity and finite element theory, including 

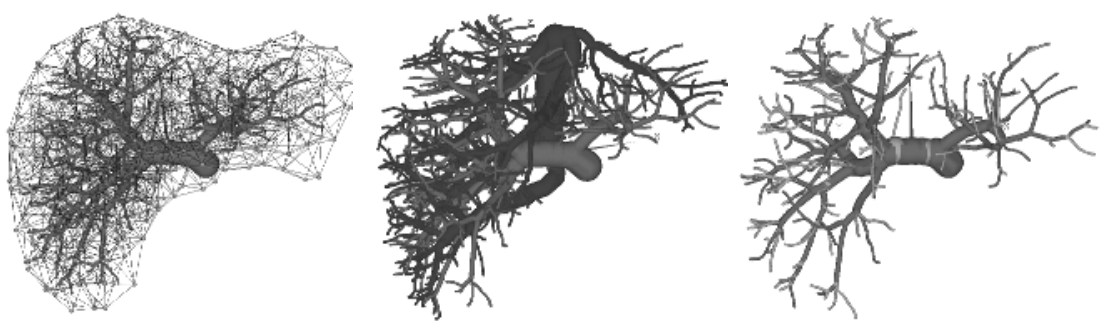

Fig. 3. The EPIDAURE surgery simulator is used to simulate liver and vascular system deformations. [Left] Volumetric model with the portal vascular system. [Center] Portal and sus-hepatic vascular system of Visible Man [Right] Portal vascular system is randomly pruned to lose approximately $40 \%$ of its branches. Lost branches appear in light gray.

anisotropic deformations. Thanks to a discussion with surgeons, we try to render realistic deformations by simulating pressure applied on the liver (breathing, patient positions, etc). The left of Fig[5] shows an example of an applied deformation.

To simulate segmentation errors, we have pruned random tree branches. Since segmentation errors are mostly observed on small vessels, the probability to lose small vessels is greater than to lose large ones. A database of 600 patient followup cases has been generated from 5 types of deformations and 5 pruning steps $(0,10,20,30,40 \%)$ with, on each step, 20 randomly generated prunings.

\subsection{Results on a Virtual Patient}

Matching: In our previous paper [1], we have demonstrated the efficiency and robustness of our matching algorithm on standard deformations. An average of $90 \%$ of all possible matches was found in the 600 different cases, even with large pruning. The process is fast and matches 380 nodes in 10 minutes on a $1 \mathrm{GHz}$ PC. Fig. 1 shows an example of a matching process where we obtain an efficiency of $91 \%$ for a standard deformation case.
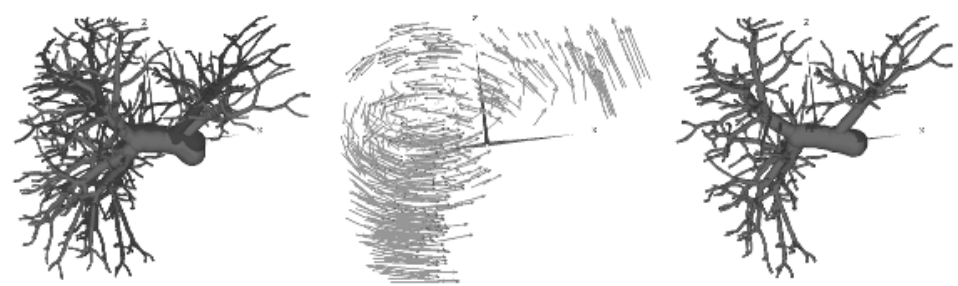

Fig. 4. [Left] Portal vascular system before the deformation estimation. [Center] Perfect match between both portal trees. [Right] Portal vascular system after the registration. 
Table 1. Deformation results on deformation n 5 : All distances are in millimeters. The liver similarity is $\frac{\operatorname{vol}\left(L_{1} \cap L_{2}\right)}{\operatorname{vol}\left(L_{1} \cup L_{2}\right)}$ with $L_{i}$ the liver area. Before the registration, the liver similarity is $\mathbf{7 2 . 3} \%$ and the mean displacement distance between livers is $\mathbf{2 2 . 7}$ $\pm \mathbf{1 0 . 3}$. With a perfect registration, these distances (errors) should be equal to 0 and liver similarity to $100 \%$.

\begin{tabular}{|c|c|c|c|c|}
\hline \% pruning & \multicolumn{2}{|c|}{ portal system } & \multicolumn{2}{c|}{ portal \& sus-hepatic system } \\
& error & similarity & error & similarity \\
\hline \hline $0-0$ & $3.0 \pm 2.0$ & 95.4 & $2.3 \pm 1.8$ & 96.4 \\
\hline $10-10$ & $3.2 \pm 2.1$ & 94.9 & $2.5 \pm 1.9$ & 96.0 \\
\hline $20-20$ & $\mathbf{3 . 9} \pm \mathbf{2 . 6}$ & $\mathbf{9 3 . 9}$ & $2.8 \pm 2.0$ & 95.6 \\
\hline $30-30$ & $4.6 \pm 3.1$ & 92.4 & $3.3 \pm 2.6$ & 94.4 \\
\hline $40-40$ & $5.0 \pm 3.3$ & 92.5 & $3.8 \pm 2.8$ & 94.0 \\
\hline $50-50$ & $5.6 \pm 3.5$ & 91.3 & $4.5 \pm 3.1$ & 92.7 \\
\hline
\end{tabular}

Table 2. Matching and deformation results: Each vascular system of these five configurations has been pruned at $20 \%$. The sensitivity (S) is the number of correct found matches among the number of perfect solution matches (here 165 nodes). The efficiency (E) is the number of correct found matches among the number of found matches (correct and incorrect). The average distance between same points in the liver are shown before registration (references), after the deformation estimated from our matching and after a deformation estimated from a perfect matching.

\begin{tabular}{|c|c|c|c|c|c|c|c|c|}
\hline cases & S & E & \multicolumn{2}{|c|}{$\begin{array}{c}\text { references } \\
\text { error }\end{array}$} & \multicolumn{2}{c|}{$\begin{array}{c}\text { found matches } \\
\text { error }\end{array}$} & \multicolumn{2}{c|}{ perfect matchilarity } \\
error & similarity \\
\hline \hline def1 & 98.8 & 90.4 & $9.9 \pm 4.2$ & 84.1 & $1.5 \pm 1.3$ & 97.0 & $1.3 \pm 1.2$ & 97.3 \\
\hline def2 & 95.5 & 87.1 & $28.9 \pm 13.6$ & 73.3 & $4.9 \pm 4.2$ & 94.2 & $4.4 \pm 3.4$ & 94.2 \\
\hline def3 & 98.2 & 86.9 & $22.9 \pm 11.8$ & 67.8 & $3.2 \pm 2.0$ & 96.0 & $3.3 \pm 2.2$ & 95.6 \\
\hline def4 & 96.0 & 87.1 & $19.7 \pm 9.8$ & 75.9 & $3.5 \pm 2.5$ & 95.3 & $3.3 \pm 2.3$ & 95.5 \\
\hline def5 & 96.1 & 87.1 & $\mathbf{2 2 . 7} \pm \mathbf{1 0 . 3}$ & 72.3 & $4.2 \pm 3.0$ & 93.6 & $\mathbf{3 . 9} \pm \mathbf{2 . 6}$ & $\mathbf{9 3 . 9}$ \\
\hline
\end{tabular}
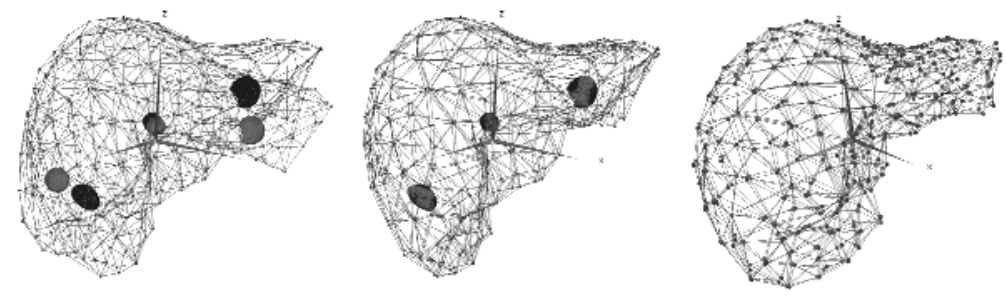

Fig. 5. [Left] Liver and its tumors before the deformation estimation. [Center] Liver and its tumors after the registration. [Right] Details on liver superimposition after the registration. 
Deformation Field: Here, we test the registration robustness only. Thus, the estimation is computed from a perfect matching. Firstly, we study the registration estimated from portal vascular system matches with different level of pruning. Secondly, we improve the estimation by adding the sus-hepatic vascular system analysis. Table 1 shows the results of these experiments. The synthetic deformation on the volumetric mesh gives us the true displacement inside the liver. Thus we compare this displacement with the one estimated from the deformation field by studying the distance between matching 3D landmarks. We compute a mean and a standard deviation on the distance between the corresponding points. According to surgeons, results are sufficient to permit a tumor follow-up: the mean error to localize a $3 \mathrm{D}$ point in the liver is $5.6 \mathrm{~mm}$ in the worst case and our registration estimation is robust against the pruning of data. Results are better when the sus-hepatic analysis is added to the process. However, the gain (about $1 \mathrm{~mm}$ ) appears small compared to the 10 additional minutes necessary to match the vascular system. Fig. 4 shows the vascular system registrations estimated from a perfect matching on the case number 5 with a $20 \%$ pruning.

\section{Conclusion}

The purpose of this paper was to present a new robust method to register the liver between two CT/MRI acquisitions using the segmented vascular systems. This registration provides us a powerful tool for the follow-up of hepatic tumors. It is easier to match tumors after this registration despite the disease evolution. Thanks to the synthetic database automatically generated by the INRIA simulator, we have tested numerous configurations. These different cases allow us to gain in robustness.

Currently, we are improving the liver deformation by testing another vector flow extrapolation. Moreover we are taking the liver surface into account to better estimate the deformation close to the surface (generally far from the vascular system). In parallel, we have started tests on a real patient database with very encouraging results (Fig. 6) and we plan to provide surgeons with a new tool for automatic diagnosis of liver tumor evolution.
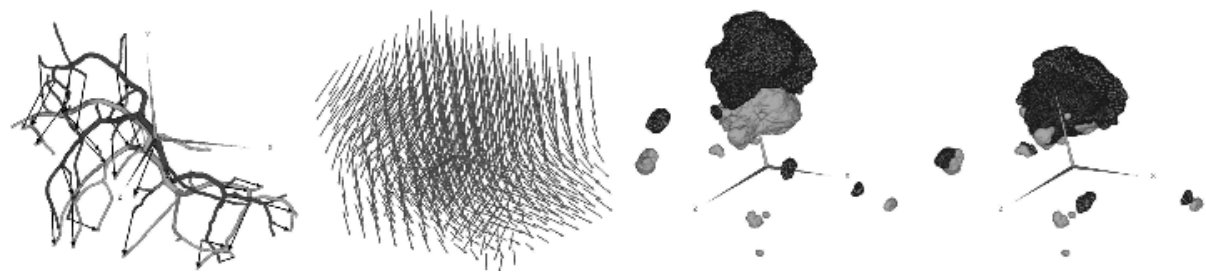

Fig. 6. [a]Real patient where the vascular system has been matched whose vertex matches are represented by black arrows. [b]Deformation field computed from matches. $[\mathbf{c}, \mathbf{d}]$ Tumors before and after registration. 
Acknowledgments. We wish to thank the Strasbourg hospital and their surgeons for providing images as well as for their advice on "standard" deformations applied to the liver. This work has benefited from the segmentation program of the vascular system developed by the IRCAD R\&D team. The realistic liver deformations are provided by the INRIA simulator from the Epidaure project. Many thanks to Clément Forest for his assistance during the use of the simulator.

\section{References}

1. Charnoz, A., Agnus, V., Malandain, G., Nicolau, S., Tajine, M., Soler, L.: Design of robust vascular tree matching: validation on liver. In: IPMI. Volume 3565 of LNCS., Glenwood Springs, Colorado, USA, Springer Verlag (2005) 443-455

2. Charnoz, A., Agnus, V., Soler, L.: Portal vein registration for the follow-up of hepatic tumours. In: MICCAI. Volume 3216 of LNCS., Saint-Malo, France, Springer Verlag (2004) 878-886

3. Aylward, S., Jomier, J., Weeks, S., Bullitt, E.: Registration and analysis of vascular images. IJCV 55 (2003) 123-138

4. Lange, T., Eulenstein, S., Hunerbein, M., Lamecker, H., Schlag, P.M.: Augmenting intraoperative 3D ultrasound with preoperative models for navigation in liver surgery. In: MICCAI. Volume 3217 of LNCS., Saint-Malo, France, Springer Verlag (2004) 534-541

5. Tschirren, J., Palágyi, K., Reinhardt, J., Hoffman, E., Sonka, M.: Segmentation, Skeletonization, and Branchpoint Matching - A Fully Automated Quantitative Evaluation of Human Intrathoracic Airway Trees. In: MICCAI. Volume 2489 of LNCS., Springer-Verlag (2002) 12-19

6. Park, Y.: Registration of linear structures in 3D medical images. PhD thesis, Osaka University, Japan. Departement of informatics and Mathematical Science (2002)

7. Pisupati, C., Wolff, L., Mitzner, W., Zerhouni, E.: Tracking 3D pulmonary tree structures. In: MMBIA, IEEE Computer Society (1996) 160

8. Soler, L., Delingette, H., Malandain, G., Montagnat, J., Ayache, N., Clément, J.M., Koehl, C., Dourthe, O., Mutter, D., Marescaux, J.: A fully automatic anatomical, pathological and fonctionnal segmentation from CT-scans for hepatic surgery. In: Medical Imaging. SPIE proceedings, San Diego (2000) 246-255

9. Pelillo, M., Siddiqi, K., Zucker, S.: Matching hierarchical structures using association graphs. PAMI 21 (1999) 1105-1120

10. Gibson, D., Spann, M.: Robust optical flow estimation based on a sparse motion trajectory set. IEEE Transactions on Image Processing (2003)

11. Cressie, N.: Statistics for Spatial Data (Revised Edition). Wiley: New York (1993)

12. Mussa-Ivaldi: From basis functions to basis fields: Vector field approximation from sparse data. In: Biological Cybernetics. Volume 67. (1992) 479-489

13. Cachier, P., Ayache, N.: Isotropic energies, filters and splines for vector field regularization. Journal of Mathematical Imaging and Vision archive 20 (2004) 147-167

14. Picinbono, G., Lombardo, J.C., Delingette, H., Ayache, N.: Improving realism of a surgery simulator: linear anisotropic elasticity, complex interactions and force extrapolation. JVCA 13 (2002) 147-167 